\title{
Comparative Study of Standard Smagorinsky Model and Dynamic Smagorinsky Model in Large Eddy Simulation of Turbulent Channel Flow
}

\author{
M. S. I. Mallik ${ }^{1 *}$, M. A. Hoque ${ }^{1,2}$, M. A. Uddin ${ }^{2}$ \\ ${ }^{1}$ Department of Arts and Sciences, Ahsanullah University of Science \& Technology, Dhaka-1208, \\ Bangladesh \\ ${ }^{2}$ Department of Mathematics, Shahjalal University of Science \& Technology, Sylhet-3114, \\ Bangladesh
}

Received 25 June 2019, accepted in final revised form 30 September 2019

\begin{abstract}
This paper presents results of comparative study of large eddy simulation (LES) that is applied to a plane turbulent channel flow. The LES is performed by using a finite difference method of second order accuracy in space and a low-storage explicit Runge-Kutta method with third order accuracy in time. In the LES for subgrid-scale (SGS) modelling, Standard Smagorinsky Model (SSM) and Dynamic Smagorinsky Model (DSM) are used. Essential turbulence statistics from the two LES approaches are calculated and compared with those from direct numerical simulation (DNS) data. Comparing the results throughout the calculation domain, it has been found out that SSM performs better than DSM in the turbulent channel flow simulation. Flow structures in the computed flow field by the SSM and DSM are also discussed and compared through the contour plots and iso-surfaces.
\end{abstract}

Keywords: Large eddy simulation; Turbulent channel flow; Standard Smagorinsky model; Dynamic Smagorinsky model.

(C) 2020 JSR Publications. ISSN: 2070-0237 (Print); 2070-0245 (Online). All rights reserved. doi: http://dx.doi.org/10.3329/jsr.v12i1.41924 J. Sci. Res. 12 (1), 39-53 (2020)

\section{Introduction}

Large eddy simulation (LES) has become a sophisticated predictive method for understanding the physics of turbulence over the few decades. The position of LES approximation is conceptually intermediate between DNS [1-3] and Reynolds-averaged Navier-Stokes (RANS) [4] techniques. Although DNS is considered as the exact approach to turbulence simulation, but it uses fine grids to resolve all the scales of motion, thus requiring a huge amount of computational resources. On the other hand, in the RANS approximation the small scales tend to depend only on viscosity, while the large ones are affected very strongly by the boundary conditions. Unlike the full-scale turbulence modeling of RANS technique, in LES method, large-scale motions are exactly calculated and the effects of subgrid-scale (SGS) motions are modeled. LES is less expensive and

\footnotetext{
*Corresponding author: saiful.austmath@gmail.com
} 
can simulate very complex flow fields in turbulence at a reasonable computational cost. So the use of LES [5-15] is increasing day by day as a reliable prediction tool.

Recent important issues for LES are SGS modeling and numerical method. There are different kinds of SGS models, such as Standard Smagorinsky model [16], Mixed Scale model [13], Dynamic models [13], etc. which are based on different relations. Different authors use different SGS models based on their simulation technique to obtain better results. With the SGS model in LES the discretization methods are also important for better accuracy of the solution. A literature review suggests that the numerical methods which are widely used for spatial discretization in LES are either spectral method or the conventional finite difference method with structured grids [17,18]. Among the two methods the finite difference method is the most straightforward one. For temporal discretization of Navier-Stokes equations explicit Runge-Kutta methods [19] are a popular choice due to their better stability properties and for adjustment with time stepping. But this method is not straightforward because of the differential-algebraic nature of equations. In wall-bounded turbulent flows for LES calculation the low storage explicit Runge-Kutta methods [20,21] have more demand among many for sufficient utilization of computer resources.

In this study, the aim is to perform LES where the SGS models used are SSM and DSM. The LES is performed in a plane turbulent channel flow. The governing equation of LES is discretized by a finite difference formulation which has a second order spatial accuracy, and for temporal discretization a low-storage explicit Runge-Kutta method with third order accuracy is applied. The performance of SSM and DSM in LES are justified through turbulence statistics and different contour plots. The statistical results are compared with the DNS data of Moser et al. [1] and Kim et al. [2]. For both of the SGS models, instantaneous vortical structures in the computed flow fields have also been discussed.

\section{Governing Equations for LES and the SGS Models}

By applying the grid filter to the dimensionless Navier-Stokes and continuity equations, the governing equations of LES for an incompressible plane turbulent channel flow in Cartesian co-ordinates are obtained as follows:

$$
\begin{aligned}
& \frac{\partial \bar{u}_{i}}{\partial t}+\frac{\partial}{\partial x_{j}}\left(\bar{u}_{i} \bar{u}_{j}+\tau_{i j}\right)=-\frac{1}{\rho} \frac{\partial \bar{p}}{\partial x_{i}}+\frac{\partial}{\partial x_{j}}\left[v\left(\frac{\partial \bar{u}_{i}}{\partial x_{j}}+\frac{\partial \bar{u}_{j}}{\partial x_{i}}\right)\right], \\
& \frac{\partial \bar{u}_{i}}{\partial x_{i}}=0
\end{aligned}
$$

where, $\bar{u}_{i}$ represents the filtered velocity component, $\bar{p}$ is the filtered pressure, $\rho$ is the fluid density and $v$ is the kinematic viscosity of the flow. The flow geometry is shown in 
Fig. 1. In the Navier-Stokes equation, $\tau_{i j}$ is the SGS Reynolds stress tensor. The effect of the SGS field, $u_{i}^{\prime}$ in LES appears through $\tau_{i j}$ which is defined by

$$
\tau_{i j}=\bar{u}_{i} u_{j}-\bar{u}_{i} \bar{u}_{j} \text {. }
$$

$\tau_{i j}$ is the unresolved term and must be modeled. The models used to resolve $\tau_{i j}$ are called SGS models. The two SGS models employed in the present study are the Standard Smagorinsky model [16] incorporating with the wall-damping function and Dynamic Smagorinsky model [22] with modification by Lilly [23]. Such a model transfers the effect of the SGS field on the filtered field.

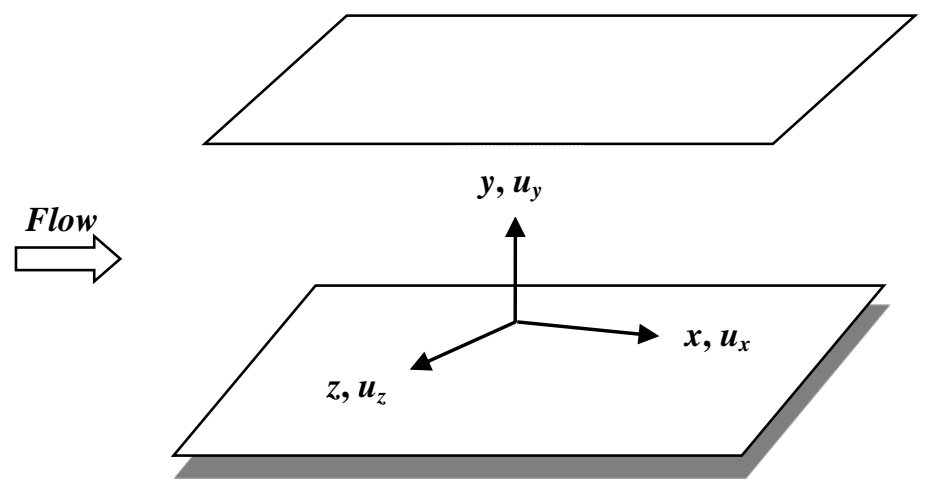

Fig. 1. Schematic geometry of plane channel flow.

\subsection{Standard Smagorinsky model (SSM)}

In this model, $\tau_{i j}$ is proportional to the filtered local strain rate tensor, $\bar{S}_{i j}$ as follows:

$$
\tau_{i j}=-2 v_{s} \bar{S}_{i j}
$$

where, $\bar{S}_{i j}=\frac{1}{2}\left(\frac{\partial \bar{u}_{i}}{\partial x_{j}}+\frac{\partial \bar{u}_{j}}{\partial x_{i}}\right)$. The proportionality factor, $v_{S}$ is the SGS eddy viscosity which is defined as

$$
v_{S}=\left(C_{S} \Delta\right)^{2}|\bar{S}|
$$

where $C_{S}$ is the Smagorinsky constant whose value is commonly used to be 0.1 for channel flow, $\Delta=(\Delta x \Delta y \Delta z)^{1 / 3}$ is the width of the grid filter, and $|\bar{S}|=\sqrt{2 \bar{S}_{i j} \bar{S}_{i j}}$ is the norm of the filtered strain rate tensor.

In this study the SSM is modified incorporating with the Van-Driest damping function, $f_{S}$ [13] in $v_{S}$ as follows: 


$$
v_{S}=\left(C_{S} f_{S} \Delta\right)^{2}|\bar{S}|
$$

where $f_{S}=1-\exp \left(-\frac{y^{+}}{A^{+}}\right)$is used to reduce the growth of small scales near the wall in which $A^{+}=25$ and $y^{+}$is the distance from the wall in viscous wall units.

\subsection{Dynamic Smagorinsky model (DSM)}

The Smagorinsky closure for $\tau_{i j}$ is given by

$$
\tau_{i j}-\frac{1}{3} \delta_{i j} \tau_{k k}=-2 v_{S} \bar{S}_{i j}
$$

where $v_{S}$ is defined in Eq. (5) and $\delta_{i j}=1$ if $i=j$ and zero otherwise. The quantity $C_{S}$ in $v_{S}$ is the Smagorinsky coefficient and $\Delta$ is the grid filter scale. In this model, the coefficient $C_{S}$ is determined locally in time and space by computing the relations given as:

$$
C_{S}^{2}=\frac{1}{2 \Delta^{2}} \frac{\left\langle M_{i j} l_{i j}\right\rangle}{\left\langle M_{i j} M_{i j}\right\rangle}
$$

where $M_{i j}=\left|\widetilde{\bar{S} \mid \bar{S}_{l j}}-\alpha^{2} \widetilde{\mid \bar{S}}\right| \widetilde{\bar{S}_{l \jmath}}, \alpha \cong \frac{\tilde{\Delta}}{\Delta}=2$,

and $l_{i j}=\left(\overline{\bar{u}_{\imath} \bar{u}_{j}}-\widetilde{\tilde{u}_{\imath}} \widetilde{\tilde{u}_{j}}\right)-\frac{1}{3} \delta_{i j}\left(\widetilde{\overline{u_{k} \bar{u}_{k}}}-\widetilde{\widetilde{u}_{k}} \widetilde{\bar{u}_{k}}\right)$,

in which a test filtering operation is indicated by tilde, $\alpha \cong \frac{\tilde{\Delta}}{\Delta}$ is the ratio of the test to grid filter widths, and " \langle\rangle ” represents an averaging operation. The test filtering is carried out with a sharp cutoff filter. Numerical tests show that an optimal value of $\alpha$ is 2 .

\section{Numerical Methods}

The numerical code is written in a staggered grid system $[9,10]$ in Cartesian coordinates. This code is based on a finite difference formulation $[9,10]$ with the second order accuracy in space and a low-storage explicit Runge-Kutta method with the third order accuracy [20] in time. The spatial and temporal schemes are shortly described in our previous papers $[9,10]$. The pressure-velocity coupling is based on the simplified markerand-cell (SMAC) method proposed by Johnson et al. [24]. In this method, the Poisson equation for pressure is solved to find the pressure potential which is used to correct the velocity components and pressure simultaneously. The Poisson equation is solved iteratively by a Preconditioned Incomplete Cholesky Decomposition Conjugated Gradient method. 


\section{Computational Parameters}

The flow domain is a rectangular box with a streamwise, wall normal and spanwise size of $2 \pi \delta, 2 \delta$ and $\pi \delta$ respectively, where $\delta$ is the channel half height. The domain is discretized by $32 \times 64 \times 32$ grid points in the corresponding size. The grid spacings in the stream-wise and span-wise directions are uniform which are $\Delta x^{+} \approx 116$ and $\Delta z^{+} \approx 58$ respectively, in wall units. In the wall normal direction $(-1 \leq y \leq+1)$, the grid spacings have been done non-uniform by stretching the grid using a hyperbolic-tangent type stretching function [25]:

$$
Y(j)=\frac{\tanh \left[\gamma\left(\frac{2 j}{N}-1\right)\right]}{\tanh (\gamma)}, j=0,1, \cdots, N,
$$

where the stretching parameter, $\gamma$ is let 2.25. In this direction, minimum grid spacing exists at the immediate vicinity of the wall which is $\Delta y^{+} \approx 2.12$ in wall unit, and the maximum grid spacing is $\Delta y^{+} \approx 42.14$ wall unit which exists at the central position of the channel. The superscript ' + ' indicates a dimensionless quantity scaled by the wall variables; e.g. $x^{+}=x u_{\tau} / v$. A sample of grid generation in the given domain is shown in Fig. 2. In this flow domain the simulation is performed at a Reynolds number, $R e_{\tau}=u_{\tau} \delta / v=590$ which is based on $\delta$ and the wall shear velocity, $u_{\tau}$. To get fully resolved turbulence scales from this simulation the computation is executed up to time, $t=n \Delta t$, where $n$ is the number of time step and $\Delta t$ is the time increment. The simulation is carried out with a non-dimensional time increment, $\Delta t=0.002$ which maintained CFL numbers $[9,10] 0.755$ and 0.817 for the LES with SSM and LES with DSM approaches respectively.

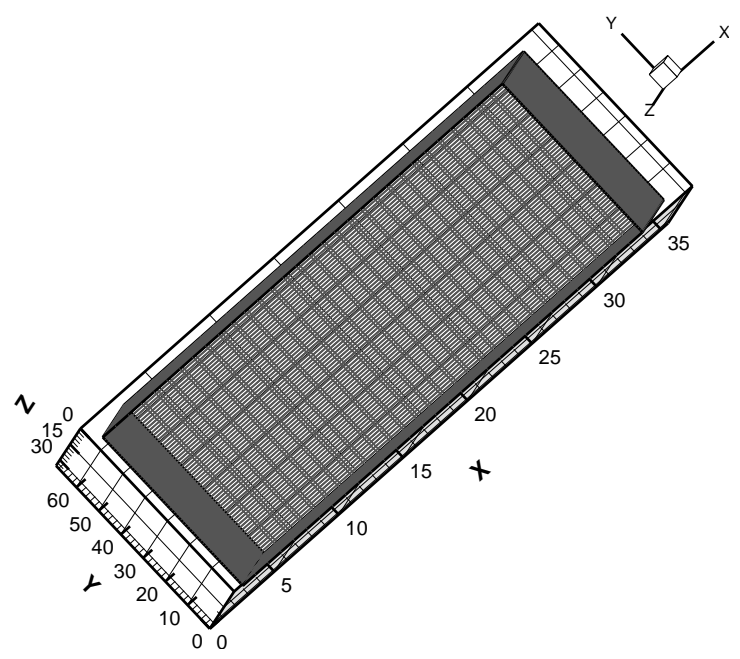

Fig. 2. A sample of grid generation in a plane channel. 


\section{Boundary Conditions}

Boundary conditions for the two LES approximations are same. Many flows that have been calculated by DNS and LES approximations allow periodic boundary conditions in one or more directions. Periodic boundary conditions are easy to implement and efficient. In this study, periodic boundary conditions are applied in the stream-wise and span-wise directions. The wall boundary condition is no-slip. In the staggered grid system, additional nodes are set up surrounding the physical boundaries. The calculations are done at the internal nodes only. At the outside nodes of the solution domain the values of the velocity components are equated to the values of the nearest node just inside the solution domain [26]. For pressure variable, the periodic boundary conditions are used in the stream-wise and span-wise directions. But, in the wall normal direction just outside the solution domain the values of pressure are determined by assuming a zero gradient [27].

\section{Results and Discussion}

\subsection{Turbulence statistics}

In this subsection we calculate some essential turbulence statistics in the given domain of the 3D turbulent channel from the computed flow fields of the two LES approximations. The initial flow field of this simulation is the random solenoidal velocity field. In a plane turbulent channel flow the boundary layer can be split mainly into two parts. One is inner or near wall region and another is outer region. Each of these regions can be divided into several layers corresponding to different types of dynamics. In the inner region, the dynamics is dominated by viscous effects and in the outer region, it is controlled by turbulence. For canonical boundary layer case, the inner region contains viscous sub-layer $\left(y^{+} \leq 5\right)$, buffer layer $\left(5<y^{+} \leq 30\right)$ and part of the logarithmic inertial layer $\left(y^{+}>30\right.$; $y / \delta<<1$ ) [13]. The outer region contains the rest of the logarithmic inertial layer and the wake region. In order to justify the performance of the two LES approximations, the statistical results are compared with DNS data of Moser et al. [1] and Kim et al. [2]. For comparison, the DNS data is represented by a solid line, LES results using SSM (LESSSM) by a dashed line and the LES results using DSM (LES-DSM) are indicated by a dashed dot dot line.

The profiles of mean velocity normalized by the wall-shear velocity corresponding to the lower half of the channel from the LES-SSM and LES-DSM approaches are shown in Fig. 3. The mean velocity is calculated by

$$
u_{x}^{+}=\frac{\left\langle\bar{u}_{x}\right\rangle}{u_{\tau}} .
$$




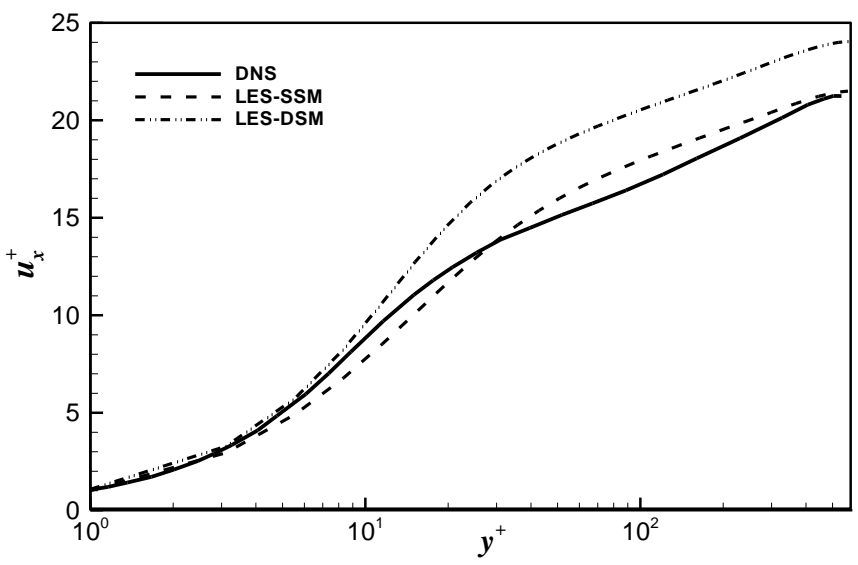

Fig. 3. Mean velocity profiles in wall units.

From this Fig. it can be observed that the LES-DSM profile over predicts the DNS profile in the whole range. Although in the viscous sub-layer and part of the buffer layer the LES-DSM profile shows less discrepancy from the DNS profile, but in rest of the range there exists a noticeable discrepancy between these two profiles. On the other hand, the LES-SSM profile is almost collapsed with the DNS profile in the viscous sub-layer. Hereafter in the buffer layer the LES-SSM profile is seen to be under predicted. Finally, in rest of the domain the LES profile over predicts the DNS profile. Nonetheless, from this figure it has to be noted that among the two LES profiles the LES-SSM profile shows closer agreement with the DNS profile in most of the region.

LES profiles of non-dimensional root mean square (r.m.s.) velocity components for SSM and DSM are displayed in Figs. 4(a, b, c). The LES profiles are compared with the DNS data in the lower half of the channel. The non-dimensional root mean square velocity components in LES can be defined as

$$
\begin{aligned}
& u_{x \text { r.m.s. }}^{+}=\sqrt{\left\langle\bar{u}_{x}^{2}\right\rangle-\left\langle\bar{u}_{x}\right\rangle^{2}} / u_{\tau}, \\
& u_{y \text { r.m.s. }}^{+}=\sqrt{\left\langle\bar{u}_{y}^{2}\right\rangle-\left\langle\bar{u}_{y}\right\rangle^{2}} / u_{\tau}, \\
& u_{z \text { r.m.s. }}^{+}=\sqrt{\left\langle\bar{u}_{z}^{2}\right\rangle-\left\langle\bar{u}_{z}\right\rangle^{2}} / u_{\tau} .
\end{aligned}
$$

Profiles of stream-wise root mean square velocity are shown in Fig. 4(a). This Fig. reveals that in the viscous sub-layer there is hardly noticeable difference between the DNS and LES profiles. After that, in rest of the range the LES profiles over predict the DNS profile which is very common in LES, because for this statistic most models will over predict the DNS data. Note that peak values of these profiles take place at the near wall region. In this region the DSM shows the largest over prediction. 

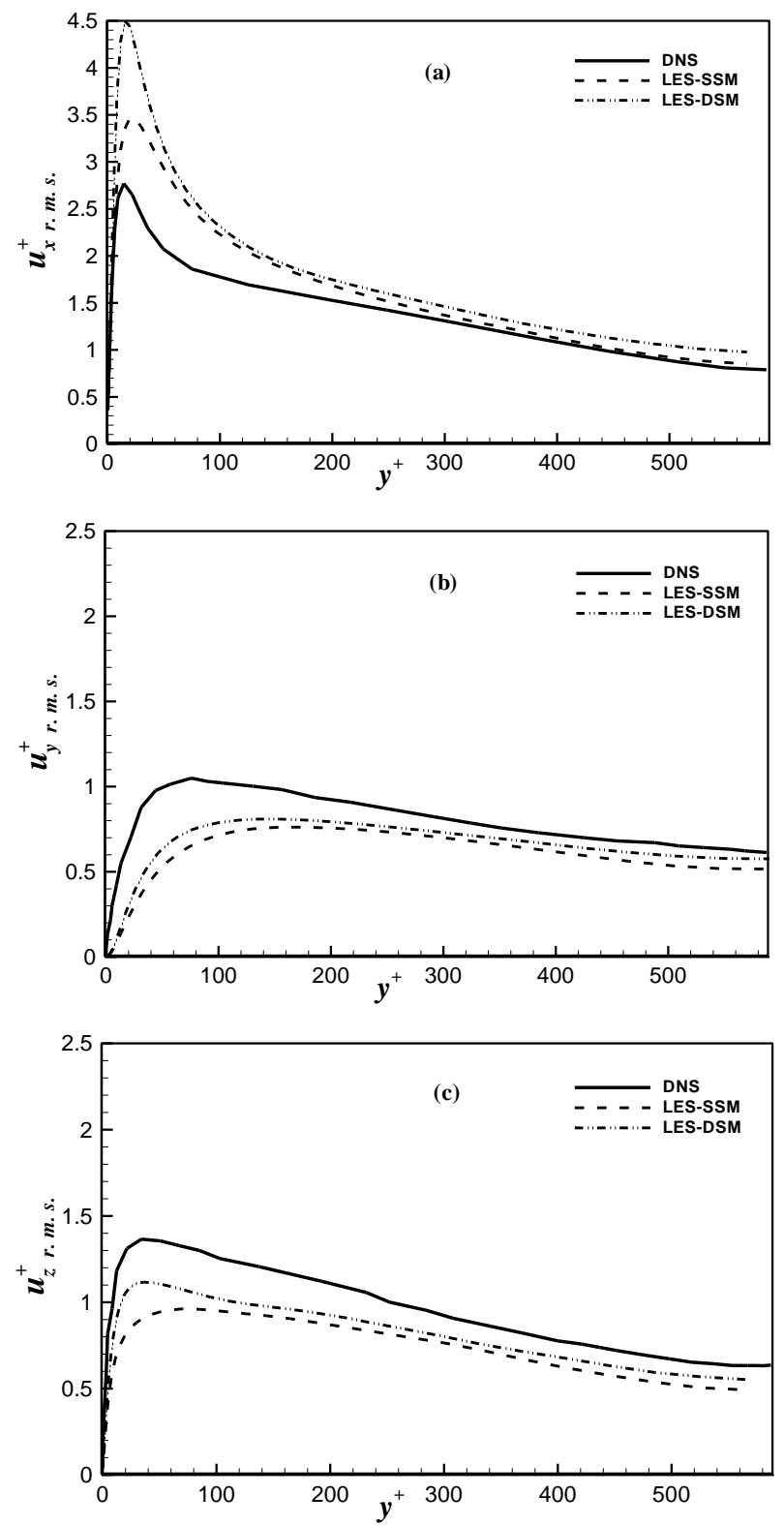

Fig. 4. Root mean square velocity profiles in wall units.

Profiles of wall normal and spanwise root mean square velocity are shown in Fig. 4(b) and Fig. 4(c) respectively. In LES it is very usual that the wall normal and spanwise root mean square velocity profiles will under predict the DNS profile. Our computed LES profiles clearly reveal that case. 


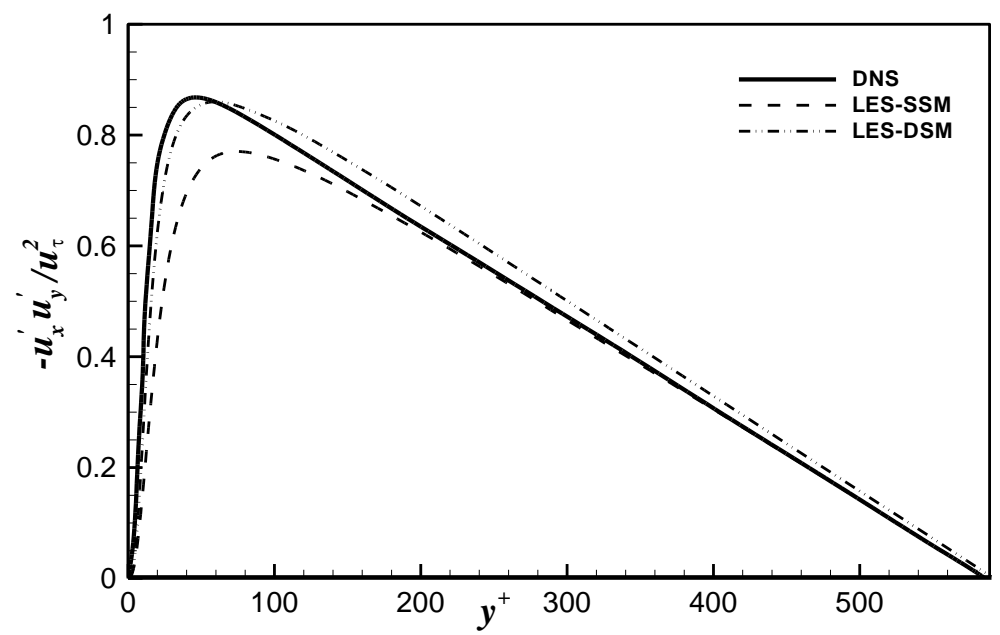

Fig. 5. Reynolds stress profiles in wall units.

Fig. 5 depicts the non-dimensional Reynolds stress, $-\frac{\overline{u_{x}^{\prime} u_{y}^{\prime}}}{{\overline{u_{\tau}^{2}}}^{2}}$ profiles for different approximations in the channel half width. From this figure it can be observed that at the near wall region the LES profiles under predict the DNS profile. On the other hand, in the outer region the LES-DSM profile over predicts the DNS profile, whereas the LES-SSM profile is almost collapsed with the DNS profile. In a fully developed channel flow this statistic appears to a straight line at an equilibrium state. Our computed LES profiles clearly maintain that case. However, among the two LES profiles the LES-SSM profile shows closer agreement with the DNS profile in most of the calculation domain. For this statistic a quantitative comparison between the DNS and LES results at some positions of the channel have been shown in the following Table.

Table 1. Comparison between DNS and LES results in Reynolds stress.

\begin{tabular}{cccc}
\hline $\boldsymbol{y}^{+}$ & DNS & LES-SSM & LES-DSM \\
\hline 4.24 & 0.08 & 0.03 & 0.04 \\
21.58 & 0.75 & 0.47 & 0.66 \\
39.06 & 0.86 & 0.69 & 0.83 \\
60.00 & 0.85 & 0.77 & 0.85 \\
101.78 & 0.80 & 0.76 & 0.82 \\
153.80 & 0.73 & 0.71 & 0.75 \\
224.21 & 0.60 & 0.59 & 0.63 \\
343.67 & 0.40 & 0.39 & 0.42 \\
449.64 & 0.22 & 0.22 & 0.24 \\
575.68 & 0.01 & 0.01 & 0.02 \\
\hline
\end{tabular}

Table 1 reveals that initially at the near wall region the LES-DSM profile shows closer agreement with the DNS profile than that of LES-SSM. At the position $y^{+} \approx 60.00$ the LES-DSM profile coincides with the DNS profile. After this position the separation of the 
LES-DSM profile from the DNS profile increases gradually up to a certain range. Then again the difference between these two profiles decreases until end of the range. On the other hand, from $y^{+} \approx 60.00$ the discrepancy of the LES-SSM profile from the DNS profile decreases gradually and after $y^{+} \approx 224.21$ these two profiles are almost collapsed. However, it is worth noting here that at the outer region the agreement of the LES-SSM profile with the DNS profile is better than that of LES-DSM.

Profiles of correlation coefficient of SGS velocity components, $u_{x}^{\prime}$ and $u_{y}^{\prime}$ corresponding to the channel full width for different approximations is presented in Fig. 6 where the correlation coefficient is defined as $\frac{-\overline{u_{x}^{\prime} u_{y}^{\prime}}}{u_{x \text { r.m.s. }} u_{y} \text { r.m.s. }}$. From this figure it can be observed that the highest value of the LES profiles appears at the lower wall, while the lowest value is located near the upper wall. The LES results for this statistic are compared with the DNS data of Kim et al. [2], where the simulation was performed in a computational domain other than the $2 \pi \delta \times 2 \delta \times \pi \delta$ with the Reynolds number, $R e_{\tau}=180$. Although, the difference between the Reynolds number of the present work and that of Kim et al. [2] is noticeable, but the pattern of the LES profiles is in good agreement with the DNS profile. In the Fig. it is important to note that among the two LES profiles the LES-SSM profile shows closer agreement with the DNS profile.

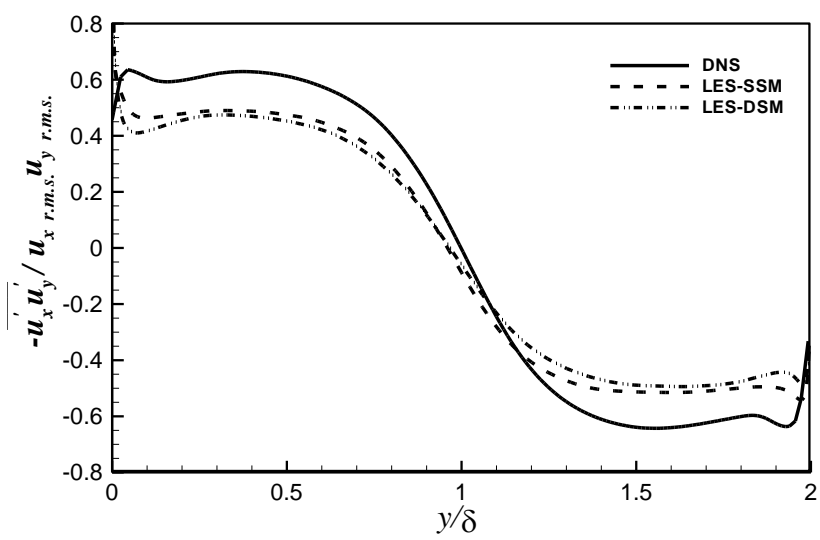

Fig. 6. Correlation coefficient profiles of $u_{x}^{\prime}$ and $u_{y}^{\prime}$.

Fig. 7 presents the profiles of SGS eddy viscosity normalized by the molecular viscosity of the flow at the stream-wise position $x=5$ of the channel for the two LES approximations. The profiles are shown corresponding to the channel full width. This Fig. reveals that the normalized $v_{S}$ starts from zero at the wall for both the approximations. The higher values of these profiles are located near the position of lower and upper walls. The value of these profiles decrease with the increase of wall units around the centerline 
of the channel. That is, the contribution of the $v_{S}$ is more near the wall position. In this figure it has to be noted that among the two LES profiles the highest peak as well as the higher values of $v_{S}$ are more located for the LES-SSM approximation than that of LESDSM.

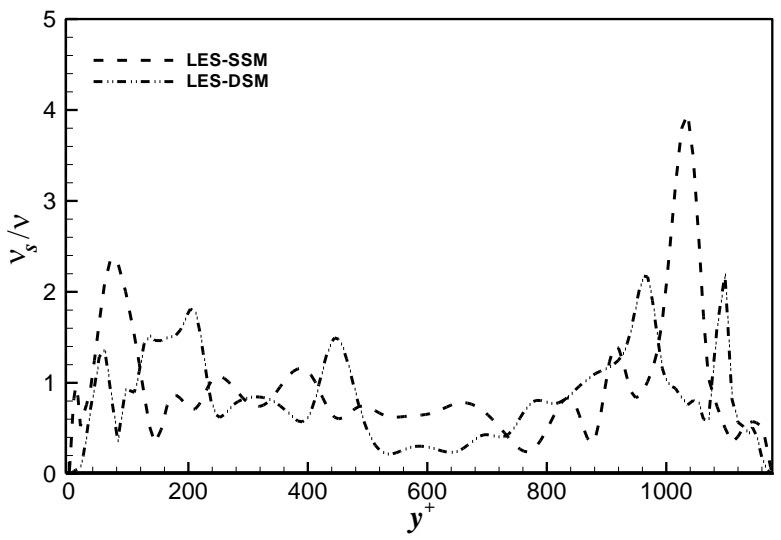

Fig. 7. SGS eddy viscosity profiles at $x=5$.

\subsection{Instantaneous distributions of stream-wise vorticity, velocity and shear velocity}

In this subsection the instantaneous distributions of the stream-wise vorticity $\left(\omega_{x}\right)$, stream-wise velocity $\left(\bar{u}_{x}\right)$ and stream-wise shear velocity $\left(\bar{u}_{x \tau}\right)$ will be discussed both for the SSM and DSM. The distributions are presented by contour plots at the end of calculation time on the $x-z$ plane. Contours of $\omega_{x}$ at the centerline $x-z$ plane of the channel are shown in Fig. 8, which is defined as

$$
\omega_{x}=\frac{\partial \bar{u}_{z}}{\partial y}-\frac{\partial \bar{u}_{y}}{\partial z}
$$
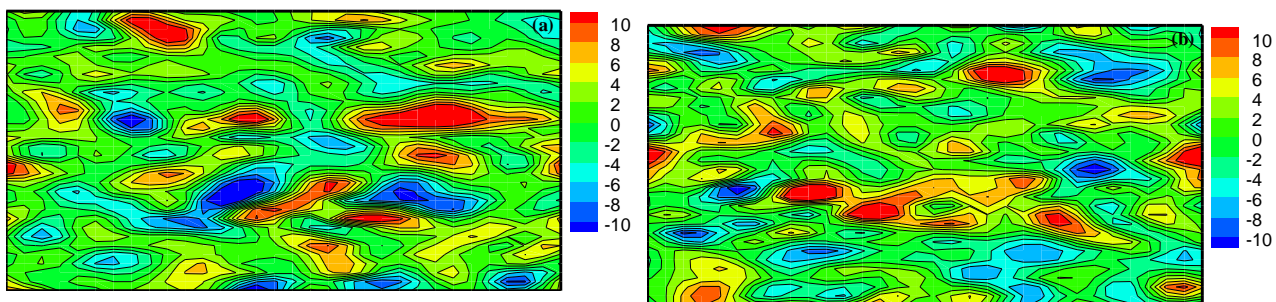

Fig. 8. Instantaneous distributions of $\omega_{x}$ in $x-z$ plane for (a) LES-SSM, and (b) LES-DSM.

It reveals that $\omega_{x}$ takes a value in the range $-10 \leq \omega_{x} \leq 10$. The highest value appears at red regions and lowest value at blue regions, but the appearance of those regions are at 
scattered locations in the whole computed flow field for both the LES approximations. It is also noticeable that in both the contour plots the contribution of the positive values of $\omega_{x}$ is more than that of the negative values. In some regions $\omega_{x}$ takes zero value. Comparing the results of $\omega_{x}$ with SSM and DSM, it can be observed that the existence of the regions of the positive values is more for DSM.
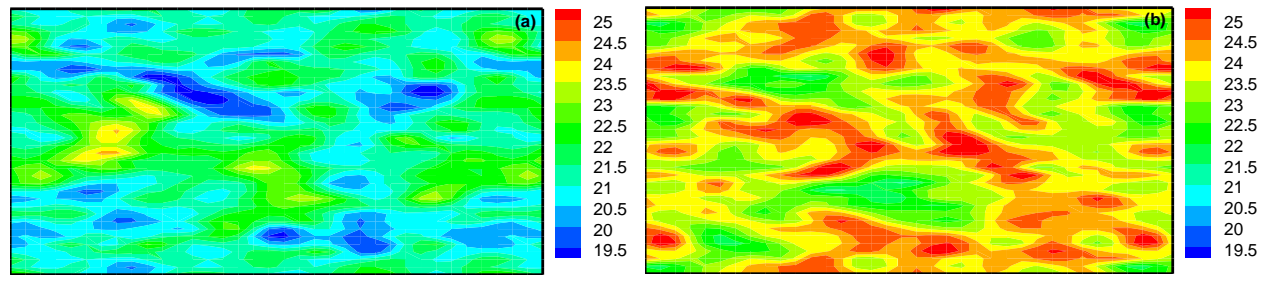

Fig. 9. Instantaneous distributions of $\bar{u}_{x}$ in $x$-z plane for (a) LES-SSM, and (b) LES-DSM.

The contours of instantaneous stream-wise velocity on the same plane for the two LES approaches are shown in Fig. 9. In this figure the values of $\bar{u}_{x}$ ranged between 19.5 and 25 . The highest value is indicated by a red color, while the lowest value by a blue color. From these contour plots, it can be observed that $\bar{u}_{x}$ takes a value in the range $19.5 \leq \bar{u}_{x} \leq 21.5$ in most of the regions in Fig. 9(a). On the other hand, in Fig. 9(b) in most of the regions $\bar{u}_{x}$ takes a value in the range $23 \leq \bar{u}_{x} \leq 25$. That is, the contribution of $\bar{u}_{x}$ is very strong for DSM. In both of the figures the appearance of the regions of the medium values of $\bar{u}_{x}$ is significant.
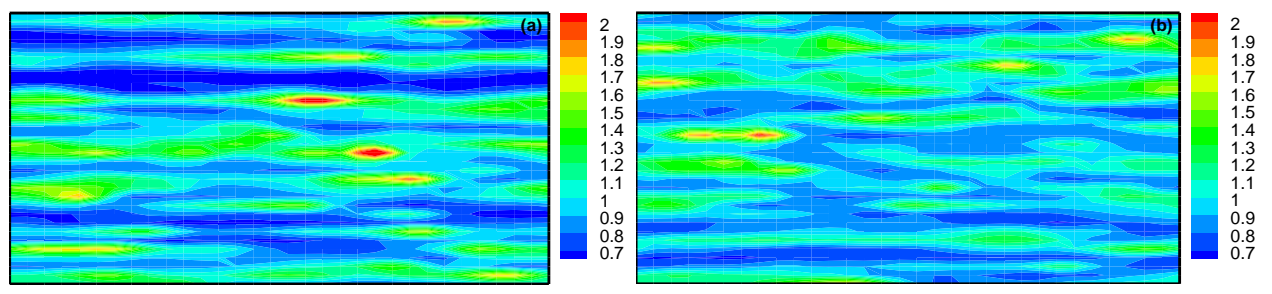

Fig. 10. Instantaneous distributions of $\bar{u}_{x \tau}$ in $x-z$ plane for (a) LES-SSM, and (b) LES-DSM.

Contours of instantaneous $\bar{u}_{x \tau}$ distribution at the immediate vicinity of the wall on the $x$ - $z$ plane both for SSM and DSM are displayed in Fig. 10, where the $\bar{u}_{x \tau}$ is calculated from Eq. (17).

$$
\bar{u}_{x \tau}=\sqrt{\frac{\tau_{x}}{\rho}},
$$

In this equation, $\rho$ is the density of the fluid and $\tau_{x}$ is the streamwise shear stress. Fig. 10 reveals that $\bar{u}_{x \tau}$ takes a value in the range $0.7 \leq \bar{u}_{x \tau} \leq 2$. The appearance of the maximum value of $\bar{u}_{x \tau}$ is not so frequent in the whole computed flow field for all the two cases, whereas, the contribution of the smaller values of $\bar{u}_{x \tau}$ is strong. It is also noticeable that 
the existence of the regions of the medium values of $\bar{u}_{x \tau}$ is significant. Comparing the results of $\bar{u}_{x \tau}$ in Fig. 10(a) and 10(b), it is noted that although the appearance of the regions of the highest and lowest value are more located in Fig. 10(a) for SSM, but the overall contribution of $\bar{u}_{x \tau}$ is more for DSM.

\subsection{Visualization of instantaneous flows}

The iso-surfaces of the second invariant, $Q$ of velocity gradient tensor at the end of computation for the two LES approaches are presented in Fig. 11. The second invariant is defined as [11]:

$$
Q=-\frac{1}{2}\left(S_{i j} S_{i j}-\Omega_{i j} \Omega_{i j}\right),
$$

where, $S_{i j}=\frac{1}{2}\left(\frac{\partial \bar{u}_{i}}{\partial x_{j}}+\frac{\partial \bar{u}_{j}}{\partial x_{i}}\right)$ and $\Omega_{i j}=\frac{1}{2}\left(\frac{\partial \bar{u}_{i}}{\partial x_{j}}-\frac{\partial \bar{u}_{j}}{\partial x_{i}}\right)$ are respectively the symmetric and asymmetric part of the velocity gradient tensor:

$$
A_{i j}=\frac{\partial u_{i}}{\partial x_{j}}=S_{i j}+\Omega_{i j}
$$
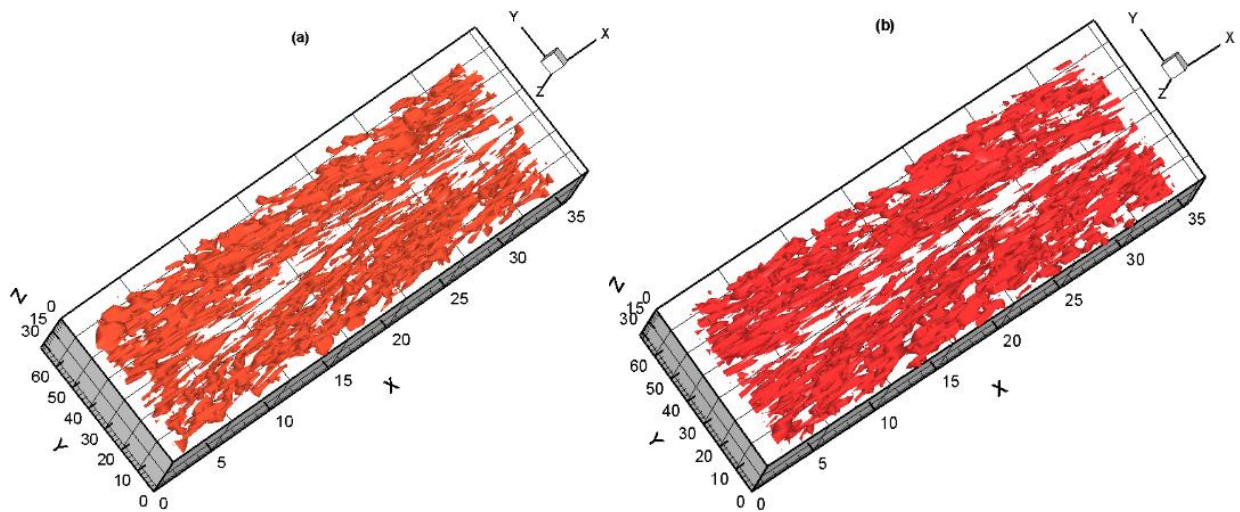

Fig. 11. Iso-surfaces of the second invariant $(Q=5)$ of velocity gradient tensor for (a) LES-SSM, and (b) LES-DSM.

The flow visualized region is the whole calculation domain and the level of the iso-surface is 5 for the both cases. This flow visualization based on $A_{i j}$ provides direct linkage between the inner and outer regions of the turbulent flow field. From this figure it can be observed that a number of tube-like vortical structures appear in the LES data both for the SSM and DSM which are randomly distributed over the turbulent flow field. But in the 
SSM case, the appearance of the vortical structures decreases from the DSM case especially near the boundary of the channel.

\section{Conclusion}

Large eddy simulations of turbulent plane channel flow have been performed using $32 \times 64 \times 32$ grid points at a Reynolds number, 590 based on the channel half width and wall shear velocity. The SGS models used for the LES simulation are the SSM and DSM. Since, in DSM the coefficient $C_{S}$ is determined locally in time and space, so DSM requires more computational cost than SSM. In order to assess the performance of the LES with SSM and DSM, essential turbulence statistics have been calculated and compared with the DNS data of reference. After analyzing the results in the statistical field we have found that in most of the cases SSM gives a good agreement with the DNS results. Although in few cases DSM performs better especially at the near wall region, but the overall performance of SSM is better than DSM, and the reason behind the less performance of DSM than SSM may be the limitations of resolution. Among the two SGS models the effect of the SGS eddy viscosity is more located for SSM especially at the near wall position. By examining the instantaneous distributions at the centerline $x$ - $z$ plane of the channel, we have observed that in the vorticity distribution the existence of the regions of positive values is more located for DSM, and in the velocity distribution the contribution of the higher values is very strong also for DSM. Instantaneous distribution of the stream-wise shear velocity at the immediate vicinity of the wall show that the appearance of the regions of the lowest and highest value is more located in the contour plot for SSM, but the overall contribution of the higher values is more for DSM. One of the distinctive features of the existence of vortical structures in the computed flow fields is that for the LES-SSM approach the vortices are generated less densely near the boundary of the channel than that of the LES-DSM approach.

\section{References}

1. R. D. Moser, J. Kim, and N. N. Mansour, Phys. Fluids 11(4), 943 (1999). https://doi.org/10.1063/1.869966

2. J. Kim, P. Moin, and R. Moser, J. Fluid Mech. 177, 133 (1987). https://doi.org/10.1017/S0022112087000892

3. M. S. I. Mallik, M. A. Uddin, and M. A. Rahman, J. Sci. Res. 5, 435 (2013). http://dx.doi.org/10.3329/jsr.v5i3.12665

4. J. H. Ferziger and M. Perić, Computational Methods for Fluid Dynamics (Springer-Verlag, Berlin, 2002). https://doi.org/10.1007/978-3-642-56026-2

5. C. D. Dritselis, Int. J. Heat Fluid Flow. 50, 225 (2014). https://doi.org/10.1016/j.ijheatfluidflow.2014.08.008

6. M. S. I. Mallik and M. A. Uddin, J. Nav. Arch. Marine Eng. 15, 75 (2018). https://doi.org/10.3329/jname.v15i2.31266

7. M. S. I. Mallik, M. A. Hoque, and M. A. Uddin, Turk. J. Math. Comp. Sci. 9, 80 (2018). http://dergipark.gov.tr/tjmcs http://tjmcs.matder.org.tr

8. M. S. I. Mallik and M. A. Uddin, J. Kor. Soc. Ind. Appl. Math. 20, 37 (2016). http://dx.doi.org/10.12941/jksiam.2016.20.037 
9. M. A. Uddin and M. S. I. Mallik, Brit. J. Math. Comp. Sci. 7, 375 (2015). https://doi.org/10.9734/BJMCS/2015/15962

10. M. S. I. Mallik, M. A. Uddin, and M. A. Meah, IOSR J. Math. 10, 41 (2014). https://doi.org/10.9790/5728-10644150

11. A. Uddin, C. Kato, Y. Yamade, N. Ohshima, M. Tanahashi, and T. Miyauchi, JSME Int. J. B 49, 102 (2006). https://doi.org/10.1299/jsmeb.49.102

12. Z. Xie, B. Lin, and R. A. Falconer, Adv. Water Resour. 53, 66 (2013). https://doi.org/10.1016/j.advwatres.2012.10.009

13. P. Sagaut, Large Eddy Simulation for Incompressible Flows: An Introduction (SpringerVerlag, Berlin, 2001). https://doi.org/10.1007/978-3-662-04416-2

14. G. Alfonsi, D. Ferraro, A. Lauria, and R. Gaudio, Phys. Fluids 31, 085105-1 (2019). https://doi.org/10.1063/1.5116522

15. M. A. E. Akhter, M. M. Alam, and M. A. K. Mallik, J. Sci. Res. 8, 129 (2016). http://dx.doi.org/10.3329/jsr.v8i2.25217

16. J. Smagorinsky, Mon. Weather Rev. 91, 99 (1963). https://doi.org/10.1175/1520-0493(1963)091<0099:GCEWTP>2.3.CO;2

17. F. E. Ham, F. S. Lien, and A. B. Strong, J. Comput. Phys. 177, 117 (2002). https://doi.org/10.1006/jcph.2002.7006

18. Y. Morinishi, J. Comput. Phys. 229, 276 (2010).

19. B. Sanderse and B. Koren, J. Comput. Phys. 231, 3041 (2012). http://dx.doi.org/10.1016/j.jcp.2011.11.028

20. C. A. Kennedy, M. H. Carpenter, and R. M. Lewis, Appl. Numer. Math. 35, 177 (2000). https://doi.org/10.1016/S0168-9274(99)00141-5

21. J. H. Williamson, J. Comput. Phys. 35, 48 (1980). https://doi.org/10.1016/0021-9991(80)90033-9

22. M. Germano, U. Piomelli, P. Moin, and W. H. Cabot, Phys. Fluids A 3, 1760 (1991). https://doi.org/10.1063/1.857955

23. D. K. Lilly, Phys. Fluids A 4, 633 (1992). https://doi.org/10.1063/1.858280

24. D. B. Johnson, P. E. Raad, and S. Chen, Int. J. Numer. Meth. Fluids 19, 153 (1994). https://doi.org/10.1002/fld.1650190205

25. Y. Morinishi, T. S. Lund, O. V. Vasilyev, and P. Moin, J. Comput. Phys. 143, 90 (1998). https://doi.org/10.1006/jcph.1998.5962

26. H. K. Versteeg and W. Malalasekera, An Introduction to Computational Fluid Dynamics (Longman Group Limited, England, 1995).

27. J. D. Jr. Anderson, Computational Fluid Dynamics (McGraw-Hill, New York, 1995). 